\title{
The spin-1/2 Heisenberg chain: thermodynamics, quantum criticality and spin-Peierls exponents
}

\author{
A. Klümper* \\ Universität zu Köln \\ Institut für Theoretische Physik \\ Zülpicher Str. 7 ry \\ D-50937, Germany
}

October 1, 2018

\begin{abstract}
We present numerical and analytical results for the thermodynamical properties of the spin-1/2 Heisenberg chain at arbitrary external magnetic field. Special emphasis is placed on logarithmic corrections in the susceptibility and specific heat at very low temperatures $\left(T / J=10^{-24}\right)$ and small fields. A longstanding controversy about the specific heat is resolved. At zero temperature the spin-Peierls exponent is calculated in dependence on the external magnetic field. This describes the energy response of the system to commensurate and incommensurate modulations of the lattice. The exponent for the spin gap in the incommensurate phase is given.
\end{abstract}

PACS : 75.10.J, 75.40, 72.15.N

Short title: "Heisenberg chain: thermodynamics, quantum criticality and instabilities"

*e-mail: kluemper@thp.uni-koeln.de 


\section{Introduction}

The seminal model for correlated quantum many-body systems is the well-known Heisenberg model. In one spatial dimension the system with nearest neighbour exchange is exactly solvable for the spin-1/2 case [1]. The elementary excitations have been classified as spinons a long time ago $[2,3]$. Despite the integrability a comprehensive theoretical treatment is still missing. A notorious problem is posed by the calculation of correlation functions even at zero temperature, not to mention the corresponding properties at finite temperature. However, the asymptotics of the correlations in particular in the groundstate and at low temperatures are quite well understood by a combination of Bethe ansatz [4] and conformal field theory [5,6]. At exactly zero temperature the spin- $1 / 2$ Heisenberg chain shows algebraic decay in its correlation functions and thereby constitutes a quantum critical system.

In recent years several experimental systems have been synthesized which realize very closely quasi one-dimensional isotropic Heisenberg chains $[7,8]$. In experimental studies the two-spinon continuum characteristic for the one-dimensional geometry has been verified in the dynamical structure factor, see for instance [9]. Some other properties probing the critical properties of the system comprise corrections to the low-temperature asymptotics in for instance the susceptibility [10]. Typically these corrections are logarithmic singularities $[11,12,13,14]$ even without impurities. The origin of this behaviour can be traced back to the existence of marginal operators (in terms of statistical mechanics).

Another aspect of the quantum criticality of the spin-1/2 chain is its instability towards a structural transition driven by long-ranged quantum fluctuations. Such instabilities generally known as Peierls or spin-Peierls transitions were originally treated in [15] for the case of free fermions (showing only marginal instability). In these days the understanding of the truly interacting case has increased in qualitative as well as quantitative aspects $[16,17]$.

In Section 2 of this paper an approach to the thermodynamics on the basis of the physical excitations (spinons) is applied to the study of several thermodynamical properties [18] (see also the related approach by $[19,20,21,22,23,24,25,26]$ ). The main results concern the numerical and analytical calculation of the universal leading and next-leading corrections in the susceptibility. In particular the resolution of a controversy between exact [27], field theoretical [28] and numerical approaches [12,13,14,29] about the specific heat at low temperatures is presented which is absolutely free of fitting procedures in the analysis of the zero-temperature limit. Our approach permits the numerical study of extremely low-temperatures down to $T / J=10^{-24}$. Furthermore the analytic treatment reveals the origin of the logarithmic corrections in certain properties of the spinon-spinon scattering phase. In Section 3 we calculate the critical exponents of correlation functions at zero temperature in the presence of an external magnetic field. On the basis of scaling relations the spin-Peierls exponents describing the gain in magnetic energy and the opening of gaps (even in the incommensurate phase) are derived. This is expected to be the basis of a more accurate treatment of the temperature-field phase diagram of spin-Peierls systems. 


\section{Thermodynamics and logarithmic corrections}

We investigate the thermodynamical properties of the antiferromagnetic isotropic spin- $1 / 2$ Heisenberg chain

$$
H=2 J \sum_{j=1}^{L} \vec{S}_{j} \vec{S}_{j+1} .
$$

We note that the elementary excitations ("spinons") have quasi-linear energy-momentum dispersion with velocity

$$
v=\pi J
$$

The free energy per lattice site of the system is given by the following set of non-linear integral equations [18] for auxiliary functions $\mathfrak{a}$, and $\mathfrak{A}=1+\mathfrak{a}$

$$
\log \mathfrak{a}(x)=-\frac{v \beta}{\cosh x}+\phi+\int_{-\infty}^{\infty}[k(x-y) \log \mathfrak{A}(y)-k(x-y-i \pi+i \epsilon) \log \overline{\mathfrak{A}}(y)] \mathrm{d} y
$$

and the corresponding equation for $\overline{\mathfrak{a}}$, and $\overline{\mathfrak{A}}=1+\overline{\mathfrak{a}}$ is obtained from (3) by exchanging $\mathfrak{a} \leftrightarrow \overline{\mathfrak{a}}, \mathfrak{A} \leftrightarrow \overline{\mathfrak{A}}$, and $i \rightarrow-i, \phi \rightarrow-\phi$. Finally, $\phi$ is given by the external magnetic field $h$ through $\phi=\beta h / 2$. The integration kernel is defined by the Fourier integral

$$
k(x)=\frac{1}{2 \pi} \int_{0}^{\infty} \frac{\mathrm{e}^{-\frac{\pi}{2} \mathrm{k}}}{\cosh \frac{\pi}{2} k} \cos (k x) d k .
$$

In terms of the solution $\mathfrak{A}$ and $\overline{\mathfrak{A}}$ to the integral equations the free energy is given by

$$
\beta f=\beta e_{0}-\frac{1}{2 \pi} \int_{-\infty}^{\infty} \frac{\log [\mathfrak{A} \overline{\mathfrak{A}}(x)]}{\cosh x} d x .
$$

These equations are readily solved numerically for arbitrary fields and temperatures by utilizing the difference type of the integral kernel and Fast Fourier Transform. In Fig. 1 the specific heat $c(T)$, susceptibility $\chi(T)$ and correlation length $\xi(T)$ (computed on the basis of [18]) at zero field are presented. Note the divergence of $\xi(T)$ at low temperatures. In Figs. 8 and 3 the results for the specific heat and susceptibility are shown for various magnetic fields below as well as above the saturation field $h_{c}=4 J$. Some qualitative aspects of the curves in dependence on the fixed external field can be understood in the picture of spinon excitations. The external magnetic field acts very much like a chemical potential for the spinons for which there are particle and hole like excitations. At zero field the bands of the particle and the hole type excitations are identical, however for sufficiently strong field $h$ the band widths are considerably different resulting into two maxima in the specific heat at different temperatures.

In Fig. $⿴$ (a) the susceptibility $\chi(T)$ is plotted down to much lower temperatures showing the onset of an infinite slope at $T=0$ as noticed in [11]. In Fig. 团 (b) a low temperature analysis of $\chi(T)$ down to $T / J=10^{-24}$ is presented giving numerical evidence about leading and subleading logarithmic corrections in temperature. Here we like to present a completely analytical low-temperature analysis (for $h<<T$ ) and defer comments about the numerical analysis to later. 


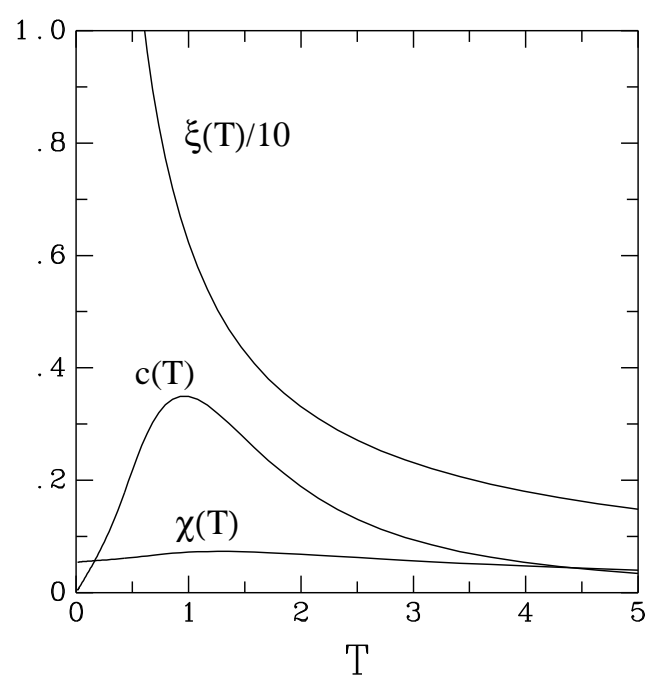

Figure 1: Plots of the specific heat $c(T)$, susceptibility $\chi(T)$ (in units of $1 / J$ ) and correlation length $\xi(T)$ at zero external field versus $T$ (in units of $J$ ).

\section{Analytical study of low-temperature asymptotics}

Because of the driving term on the RHS of (3) it is convenient to introduce the following scaling functions

$$
\begin{aligned}
\mathfrak{a}_{\mathfrak{L}}(x) & =\mathfrak{a}(x+\mathfrak{L}), \quad \mathfrak{L}=\log (\pi \beta), \\
\widetilde{\mathfrak{a}}_{\mathfrak{L}}(x) & =\mathfrak{a}(-x-\mathfrak{L}),
\end{aligned}
$$

approaching well-defined non-trivial limiting functions in the low-temperature limit which satisfy

$$
\begin{aligned}
\log \mathfrak{a}_{\mathfrak{L}}(x)= & -2 \mathrm{e}^{-\mathrm{x}}+\mathrm{O}(1 / \beta)+\phi+\psi_{\mathfrak{L}}(\mathrm{x}) \\
& +\int_{-\mathfrak{L}}^{\infty}\left[k(x-y) \log \mathfrak{A}_{\mathfrak{L}}(y)-k(x-y-i \pi+i \epsilon) \log \overline{\mathfrak{A}}_{\mathfrak{L}}(y)\right] \mathrm{d} y
\end{aligned}
$$

where $\psi_{\mathfrak{L}}(x)$ accounts for the contribution of the functions $\mathfrak{A}$ and $\overline{\mathfrak{A}}$ on the negative real axis

$$
\psi_{\mathfrak{L}}(x)=\int_{-\mathfrak{L}}^{\infty}\left[k(x+y+2 \mathfrak{L}) \log \widetilde{\mathfrak{A}}_{\mathfrak{L}}(y)-k(x+y+2 \mathfrak{L}-i \pi+i \epsilon) \log \widetilde{\overline{\mathfrak{A}}}_{\mathfrak{L}}(y)\right] \mathrm{d} y .
$$

Using these functions the free energy at low temperatures takes the form

$$
f=e_{0}-\frac{1}{\pi v \beta^{2}} \int_{-\mathfrak{L}}^{\infty} \mathrm{e}^{-\mathrm{x}} \log \left[\mathfrak{A}_{\mathfrak{L}} \overline{\mathfrak{A}}_{\mathfrak{L}} \widetilde{\mathfrak{A}}_{\mathfrak{L}} \widetilde{\overline{\mathfrak{A}}}_{\mathfrak{L}}(\mathrm{x})\right] \mathrm{dx}+\mathrm{O}\left(1 / \beta^{3}\right)
$$



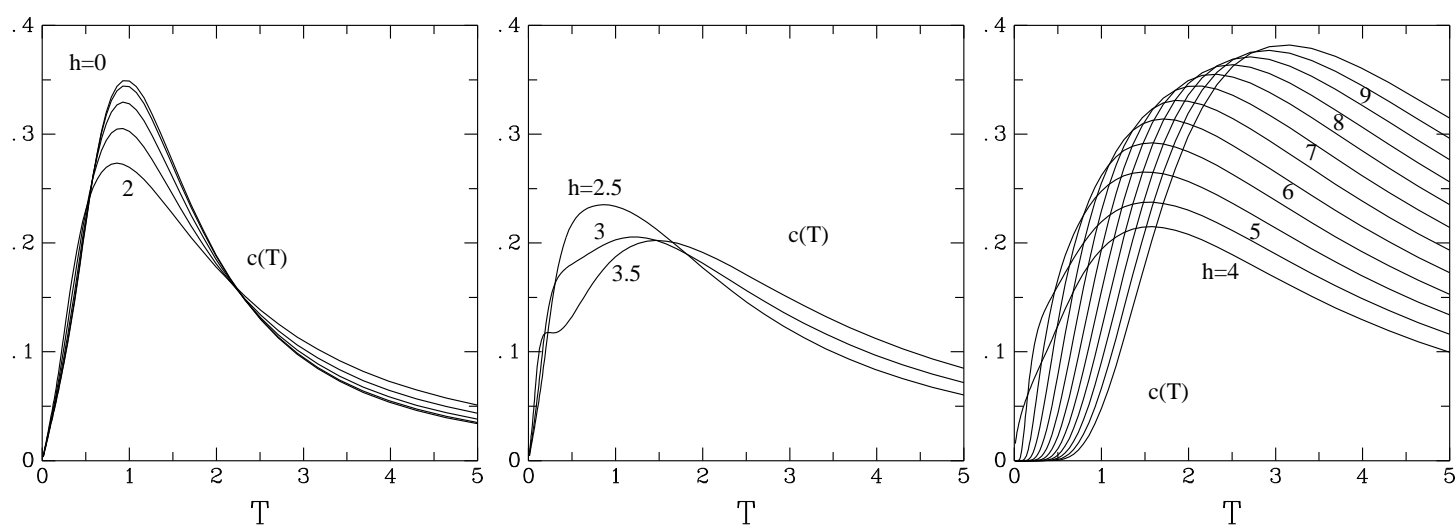

Figure 2: Depiction of the specific heat of the Heisenberg chain for different values of the magnetic field (a) $h / J=0,0.5, \ldots, 2.0$, (b) $h / J=2.5, \ldots, 3.5$, (c) $h / J=4.0, \ldots, 9.5$. Note the linear $T$ dependence at low temperatures $T$ for fields less than the saturation value $h_{c} / J=4$, see (a) and (b). For $h=h_{c}$ the specific heat is proportional to $T^{1 / 2}$ for sufficiently low $T$, and for even stronger fields a thermodynamically activated behaviour develops, see (c).

In order to analyse the low-temperature asymptotics in detail we perform the following manipulation

$$
\begin{aligned}
& \int_{-\mathfrak{L}}^{\infty}\left[\left(\log \mathfrak{a}_{\mathfrak{L}}\right)^{\prime}\left(\log \mathfrak{A}_{\mathfrak{L}}\right)-\left(\log \mathfrak{a}_{\mathfrak{L}}\right)\left(\log \mathfrak{A}_{\mathfrak{L}}\right)^{\prime}\right] d x+{ }^{'} \operatorname{conj}, \\
& =\int_{-\mathfrak{L}}^{\infty}\left[\left(-2 \mathrm{e}^{-\mathrm{x}}+\phi+\psi_{\mathfrak{L}}(\mathrm{x})\right)^{\prime}\left(\log \mathfrak{A}_{\mathfrak{L}}\right)-\left(-2 \mathrm{e}^{-\mathrm{x}}+\phi+\psi_{\mathfrak{L}}(\mathrm{x})\right)\left(\log \mathfrak{A}_{\mathfrak{L}}\right)^{\prime}\right] d x+{ }^{\prime} \operatorname{conj}{ }^{\prime} \\
& =2 \int_{-\mathfrak{L}}^{\infty}\left(2 \mathrm{e}^{-\mathrm{x}}+\psi_{\mathfrak{L}}(\mathrm{x})^{\prime}\right)\left(\log \mathfrak{A}_{\mathfrak{L}}\right) \mathrm{dx}-\phi \log \left(1+\mathrm{e}^{2 \phi}\right)+{ }^{\prime} \operatorname{conj} ;
\end{aligned}
$$

where in the second line we have inserted (7) and in the third line we have performed an integration by parts with an explicit evaluation of the contribution by the integral terminals using the limits

$$
\begin{array}{rlrl}
\log \mathfrak{a}_{\mathfrak{L}}(\infty) & =+2 \phi, & & \log \mathfrak{A}_{\mathfrak{L}}(\infty)=\log \left(1+\mathrm{e}^{+2 \phi}\right) \\
\log \overline{\mathfrak{a}}_{\mathfrak{L}}(\infty)=-2 \phi, & \log \overline{\mathfrak{A}}_{\mathfrak{L}}(\infty)=\log \left(1+\mathrm{e}^{-2 \phi}\right)
\end{array}
$$



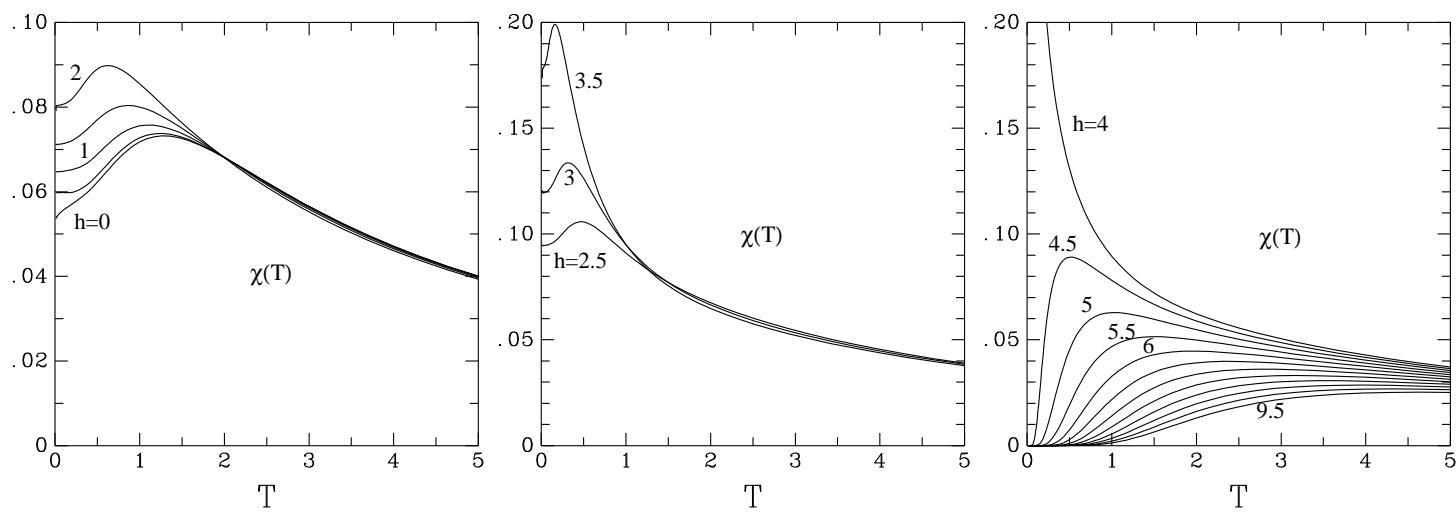

Figure 3: Depiction of the susceptibility (in units of $1 / J$ ) of the Heisenberg chain for different values of the magnetic field (a) $h / J=0,0.5, \ldots, 2.0$, (b) $h / J=2.5, \ldots, 3.5$, (c) $h / J=4.0, \ldots, 9.5$. Note the finite value at zero temperature for fields less than the saturation value $h_{c} / J=4$, see (a) and (b). For $h=h_{c}$ the susceptibility diverges at $T=0$, and for fields larger than $h_{c}$ the susceptibility drops to zero, see (c).

Next, we show that the LHS of (10) can be evaluated explicitly as it is a definite integral of dilogarithmic type with known terminals

$$
\begin{aligned}
L H S & =2 \int_{-\mathfrak{L}}^{\infty}\left(\log \mathfrak{a}_{\mathfrak{L}}\right)^{\prime} \log \mathfrak{A}_{\mathfrak{L}} d x-\left.\log \mathfrak{a}_{\mathfrak{L}}\left(\log \mathfrak{A}_{\mathfrak{L}}\right)^{\prime}\right|_{-\mathfrak{L}} ^{\infty}+{ }^{\prime} \operatorname{conj}{ }^{\prime} \\
& =2 \int_{-\infty}^{2 \phi} \log \left(1+\mathrm{e}^{\mathrm{z}}\right) \mathrm{dz}-2 \phi \log \left(1+\mathrm{e}^{2 \phi}\right)+2 \int_{-\infty}^{-2 \phi} \log \left(1+\mathrm{e}^{\mathrm{z}}\right) \mathrm{dz}+2 \phi \log \left(1+\mathrm{e}^{-2 \phi}\right) \\
& =\frac{\pi^{2}}{3},
\end{aligned}
$$

and the final result for the definite integral in the second line was obtained by standard techniques (it is independent of $\phi$ and the particular choice $\phi=0$ "directly" leads to the final result).

Combining (10) and (12) we arrive at a formula particularly suited for studying the asymptotic behaviour of (9)

$$
4 \int_{-\mathfrak{L}}^{\infty} \mathrm{e}^{-\mathrm{x}} \log \left[\mathfrak{A}_{\mathfrak{L}} \overline{\mathfrak{A}}_{\mathfrak{L}}\right] \mathrm{dx}=\frac{\pi^{2}}{3}+2 \phi^{2}-2 \int_{-\mathfrak{L}}^{\infty}\left[\psi_{\mathfrak{L}}(\mathrm{x})^{\prime} \log \mathfrak{A}_{\mathfrak{L}}+\bar{\psi}_{\mathfrak{L}}(\mathrm{x})^{\prime} \log \overline{\mathfrak{A}}_{\mathfrak{L}}\right] \mathrm{dx} .
$$

From the general expression of the free energy $f(5)$ in terms of the functions $\mathfrak{A}$ and $\overline{\mathfrak{A}}$ the entire functional dependence of $\mathfrak{A}$ and $\overline{\mathfrak{A}}$ matters even for the calculation of the asymptotic behaviour of $f$ at low temperature. The usefulness of (13) lies in the fact that here only the asymptotic behaviour of $\mathfrak{A}$ and $\overline{\mathfrak{A}}$ at large spectral parameter enters. The reason is simple to understand: for any finite argument $x$ the contribution is of order $1 / \mathfrak{L}^{2}$ due to the $\psi_{\mathfrak{L}}(x)$ factors. Here we see that the low-temperature asymptotics (and further below the occurrence of logarithmic corrections) are intimately linked to the algebraic 

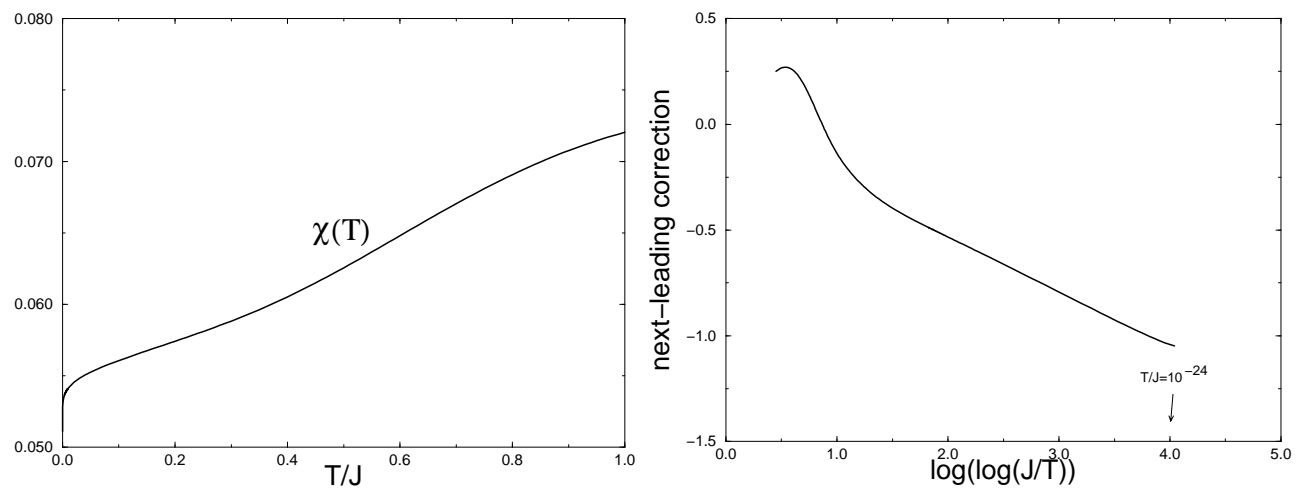

Figure 4: Plots of (a) zero-field susceptibility $\chi(T)$ versus $T / J$ showing a logarithmic singularity at $T=0$ and (b) the next-leading logarithmic correction $\left(\chi / \chi_{0}-1-1 / 2 \mathfrak{L}\right) \mathfrak{L}^{2}$ versus $\log \mathfrak{L}(\simeq \log \log J / T)$ down to $T / J=10^{-24}$. Note the linear dependence on $\log \mathfrak{L}$ for low temperatures.

decay of the integration kernel $k(x)$

$$
k(x) \simeq \frac{1}{\pi^{2}+4 x^{2}} .
$$

We like to add that the kernel is related to the $S$-matrix of the elementary spinons [3] by $k(x)=\frac{d}{d x} \log S(x)$.

\section{Asymptotics of the susceptibility}

In a first step we determine the asymptotics of the auxiliary functions from (7) where we drop integrations over negative arguments and for large arguments we use $\overline{\mathfrak{a}}_{\mathfrak{L}} \simeq 1 / \mathfrak{a}_{\mathfrak{L}}$ (hence $\log \mathfrak{A}_{\mathfrak{L}}-\log \overline{\mathfrak{A}}_{\mathfrak{L}}=\log \mathfrak{a}_{\mathfrak{L}}$ ) and furthermore we equate the kernels $k(\ldots)$ with arguments differing by $\pi i$ leading to

$$
\begin{aligned}
\log \mathfrak{a}_{\mathfrak{L}}(x)= & \phi+\psi_{\mathfrak{L}}(x)+\int_{0}^{\infty} k(x-y) \log \mathfrak{a}_{\mathfrak{L}}(y) \mathrm{d} y \\
= & \phi+\psi_{\mathfrak{L}}(x)+\int_{0}^{\infty} k(x-y) \log \frac{\mathfrak{a}_{\mathfrak{L}}(y)}{\mathfrak{a}_{\mathfrak{L}}(\infty)} \mathrm{d} y+\int_{0}^{\infty} k(x-y) \log \mathfrak{a}_{\mathfrak{L}}(\infty) \mathrm{d} y \\
= & \phi+\psi_{\mathfrak{L}}(x)+\log \frac{\mathfrak{a}_{\mathfrak{L}}(x)}{\mathfrak{a}_{\mathfrak{L}}(\infty)} \int_{0}^{\infty} k(x-y) \mathrm{d} y \\
& +\int_{-\infty}^{\infty} k(x-y) \log \mathfrak{a}_{\mathfrak{L}}(\infty) \mathrm{d} y-\int_{-\infty}^{0} k(x-y) \log \mathfrak{a}_{\mathfrak{L}}(\infty) \mathrm{d} y \\
= & \phi+\psi_{\mathfrak{L}}(x)+\frac{1}{2} \log \mathfrak{a}_{\mathfrak{L}}(x)-\int_{-\infty}^{0} \frac{\log \mathfrak{a}_{\mathfrak{L}}(y)}{4(x-y)^{2}} \mathrm{~d} y \\
= & \phi+\psi_{\mathfrak{L}}(x)+\frac{1}{2} \log \mathfrak{a}_{\mathfrak{L}}(x)-\frac{\phi}{2 x}
\end{aligned}
$$


In the first integral of the second line we have used the fact that dominant contributions occur for the integration variable $y$ close to $x$. In the subsequent calculations the asymptotic behaviour of the kernel and also the total integral $\int k(x) d x=1 / 2$ were used. Finally, employing the limits (11) we arrive at

$$
\log \mathfrak{a}_{\mathfrak{L}}(x)=2 \phi-\frac{\phi}{x} .
$$

In the second step we insert (8) into the integral on the RHS of (13), then we insert (16) and calculate the resulting integral by dropping terms leading to contributions of order $O\left(1 / \mathfrak{L}^{2}\right)$ and higher

$$
\begin{aligned}
& \int_{-\mathfrak{L}}^{\infty}\left[\psi_{\mathfrak{L}}(x)^{\prime} \log \mathfrak{A}_{\mathfrak{L}}+\bar{\psi}_{\mathfrak{L}}(x)^{\prime} \log \overline{\mathfrak{A}}_{\mathfrak{L}}\right] d x \\
& =+4 \phi^{2} \int_{1}^{\infty} \int_{1}^{\infty} k^{\prime}(x+y+2 \mathfrak{L})\left(1-\frac{1}{2 x}\right)\left(1-\frac{1}{2 y}\right) d x d y \\
& =-4 \phi^{2} \int_{1}^{\infty} k(x+2 \mathfrak{L})\left(1-\frac{1}{x}\right) d x=-\phi^{2} \int_{1}^{\infty} \frac{1}{(x+2 \mathfrak{L})^{2}}\left(1-\frac{1}{x}\right) d x \\
& =\phi^{2}\left(-\frac{1}{2 \mathfrak{L}}+\frac{\log (2 \mathfrak{L})}{(2 \mathfrak{L})^{2}}\right) .
\end{aligned}
$$

Hence we obtain the dependence of the free energy on the magnetic field at low temperature

$$
4 \int_{-\mathfrak{L}}^{\infty} \mathrm{e}^{-\mathrm{x}} \log \left[\mathfrak{A}_{\mathfrak{L}} \overline{\mathfrak{A}}_{\mathfrak{L}}\right] \mathrm{dx}=\frac{\pi^{2}}{3}+2 \phi^{2}\left(1+\frac{1}{2 \mathfrak{L}}-\frac{\log (2 \mathfrak{L})}{(2 \mathfrak{L})^{2}}\right),
$$

implying logarithmic corrections in $T$ for the free energy and the zero-field susceptibility

$$
\begin{aligned}
f & =e_{0}-\frac{\pi}{6 v} T^{2}-\frac{h^{2}}{4 \pi v}\left(1+\frac{1}{2 \mathfrak{L}}-\frac{\log (2 \mathfrak{L})}{(2 \mathfrak{L})^{2}}\right), \quad \mathfrak{L}=\log (\pi / T), \quad(h<<T), \\
\chi & =\frac{1}{2 \pi v}\left(1+\frac{1}{2 \mathfrak{L}}-\frac{\log (2 \mathfrak{L})}{(2 \mathfrak{L})^{2}}\right) .
\end{aligned}
$$

This result is in complete agreement with the numerical analysis in Fig. (1) (b) and with $[30,29]$ (if the system size $L$ is replaced by the inverse temperature $1 / T$ ). The (nonuniversal) temperature scale $T_{0}$ entering the logarithms of the correction terms will be studied elsewhere [31].

\section{Asymptotics of the specific heat}

Next we calculate the higher order low-temperature asymptotics of the Heisenberg chain in zero external magnetic field. Here the functions $\mathfrak{a}_{\mathfrak{L}}$ and $\overline{\mathfrak{a}}_{\mathfrak{L}}$ are related by complex conjugation. We therefore write

$$
\begin{aligned}
\log \mathfrak{A}_{\mathfrak{L}}(x) & =\log \widetilde{\overline{\mathfrak{A}}}_{\mathfrak{L}}(x)=R_{\mathfrak{L}}(x)+i I_{\mathfrak{L}}(x), \\
\log \overline{\mathfrak{A}}_{\mathfrak{L}}(x) & =\log \widetilde{\mathfrak{A}}_{\mathfrak{L}}(x)=R_{\mathfrak{L}}(x)-i I_{\mathfrak{L}}(x) .
\end{aligned}
$$


Again we insert (8) into the integral on the RHS of (13), then we insert (21) and expand $k(x+\pi i)=k(x)+k^{\prime}(x) \pi i-k^{\prime \prime}(x) \pi^{2} / 2$ obtaining

$$
\begin{aligned}
& \int_{-\mathfrak{L}}^{\infty}\left[\psi_{\mathfrak{L}}(x)^{\prime} \log \mathfrak{A}_{\mathfrak{L}}+\bar{\psi}_{\mathfrak{L}}(x)^{\prime} \log \overline{\mathfrak{A}}_{\mathfrak{L}}\right] d x \\
& =\int_{-\mathfrak{L}}^{\infty} \int_{-\mathfrak{L}}^{\infty} d x d y\left[\pi^{2} k^{\prime \prime \prime}(x+y+2 \mathfrak{L}) R_{\mathfrak{L}}(x) R_{\mathfrak{L}}(y)+4 k^{\prime}(x+y+2 \mathfrak{L}) I_{\mathfrak{L}}(x) I_{\mathfrak{L}}(y)\right. \\
& \left.\quad-2 \pi k^{\prime \prime}(x+y+2 \mathfrak{L})\left(R_{\mathfrak{L}}(x) I_{\mathfrak{L}}(y)+I_{\mathfrak{L}}(x) R_{\mathfrak{L}}(y)\right)\right] \\
& =\int_{-\mathfrak{L}}^{\infty} \int_{-\mathfrak{L}}^{\infty} d x d y\left[\pi^{2} k^{\prime}(x+y+2 \mathfrak{L}) R_{\mathfrak{L}}^{\prime}(x) R_{\mathfrak{L}}^{\prime}(y)+4 k^{\prime}(x+y+2 \mathfrak{L}) I_{\mathfrak{L}}(x) I_{\mathfrak{L}}(y)\right. \\
& \left.\quad+2 \pi k^{\prime}(x+y+2 \mathfrak{L})\left(R_{\mathfrak{L}}^{\prime}(x) I_{\mathfrak{L}}(y)+I_{\mathfrak{L}}(x) R_{\mathfrak{L}}^{\prime}(y)\right)\right]
\end{aligned}
$$

where in the last step we have performed an integration by parts. Next, the integral is dominated by contributions with finite integration variable $x$ and $y$ for which $k^{\prime}(\ldots)$ can be replaced by a constant yielding

$$
\begin{aligned}
R H S & =-\frac{1}{16 \mathfrak{L}^{3}} \int_{-\mathfrak{L}}^{\infty} \int_{-\mathfrak{L}}^{\infty} d x d y\left[\pi R_{\mathfrak{L}}^{\prime}(x)+2 I_{\mathfrak{L}}(x)\right]\left[\pi R_{\mathfrak{L}}^{\prime}(y)+2 I_{\mathfrak{L}}(y)\right], \\
& =-\frac{1}{16 \mathfrak{L}^{3}}(\pi R+2 I)^{2},
\end{aligned}
$$

where we have used the abbreviations

$$
\begin{aligned}
R & =\int_{-\mathfrak{L}}^{\infty} d x R_{\mathfrak{L}}^{\prime}(x)=\log 2 \\
I & =\int_{-\mathfrak{L}}^{\infty} d x I_{\mathfrak{L}}(x) \rightarrow \text { numerical evaluation, }
\end{aligned}
$$

where $R$ can be calculated easily from the asymptotics of $R_{\mathfrak{L}}(x), I$ however has to be evaluated by numerical calculations. For the free energy we obtain

$$
4 \int_{-\mathfrak{L}}^{\infty} \mathrm{e}^{-\mathrm{x}} \log \left[\mathfrak{A}_{\mathfrak{L}} \overline{\mathfrak{A}}_{\mathfrak{L}}\right] \mathrm{d} \mathrm{x}=\frac{\pi^{2}}{3}\left[1+\frac{3}{8}\left(\mathrm{R}+\frac{2}{\pi} \mathrm{I}\right)^{2} \frac{1}{\mathfrak{L}^{3}}\right],
$$

where $\left(R+\frac{2}{\pi} I\right)$ is found to be $1 \pm 10^{-6}$. This leads to

$$
f=e_{0}-\frac{\pi}{6 v} T^{2}\left(1+\frac{3}{8} \frac{1}{\mathfrak{L}^{3}}\right), \quad \mathfrak{L}=\log (\pi / T),
$$

confirming the field theoretical prediction in [28] about the amplitude of the $1 /(\log T)^{3}$ correction which was argued to be universal however in disagreement with Bethe ansatz calculations in [27]. The reason for the "failure" of the treatment in [27] (instead of 

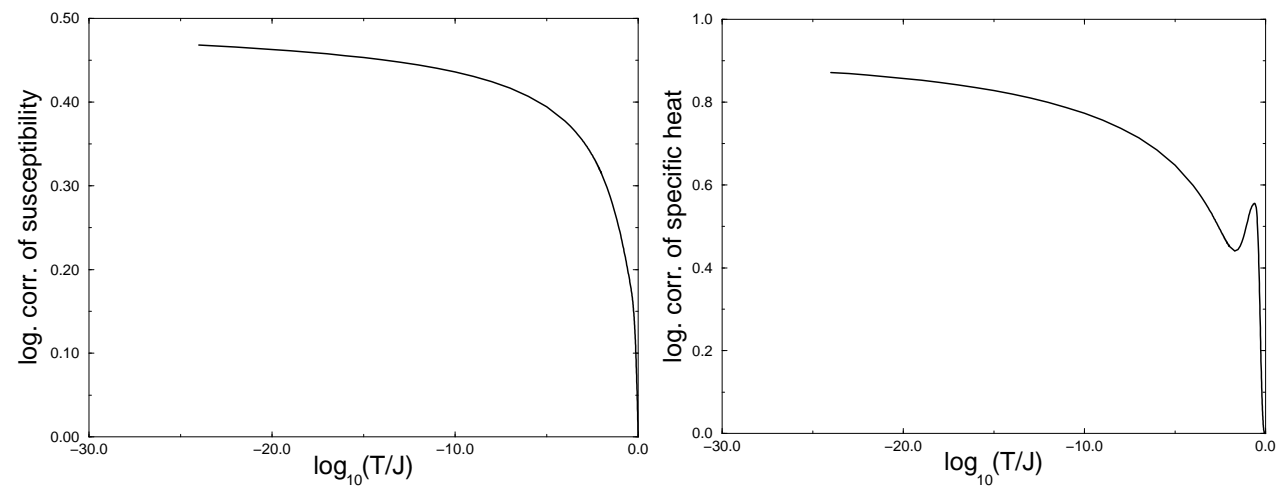

Figure 5: Plots of the estimators of the leading logarithmic corrections (a) for the susceptibility $\left(\chi / \chi_{0}-1\right) \mathfrak{L}$ and $(b)$ the specific heat $[3 v c(T) / \pi T-1] \frac{8}{3} \mathfrak{L}^{3}$ versus $\log _{10}(T / J)$. Note the non-monotonous behaviour of the higher order terms in (b).

$3 / 8$ the value 0.3433 was obtained) is subtle and lies in an inappropriate use of the Euler-MacLaurin formula which unfortunately is common practice in the treatment of critical lattice systems. The numerical treatments of the Bethe ansatz equations up to $N=16384[12,13,14]$ were still plagued by higher order logarithmic contributions leading to erroneous conclusions with respect to the analysis of the specific heat. For an illustration of these higher order terms see Fig. 5. For the case of the specific heat an appropriate fit of finite size data seems very difficult. We note, that the careful numerical treatment [29] of finite systems up $N=16384$ employed an appropriate fit algorithm taking into account the proper higher correction terms. Our results agree with the conclusions of [29]. We emphasize that our treatment is completely free of any fit procedures. The type of logarithmic singularities has been derived analytically and the amplitudes have been calculated (in the worst case) numerically which did not face any extrapolation problems.

\section{Groundstate properties of homogeneous and dimerized $\mathrm{S}=1 / 2$ Heisenberg chains}

Next we treat the critical groundstate properties of the homogeneous Heisenberg chain by means of an application of the exact Bethe ansatz solution and conformal field theory, see [32] and references therein. 


\section{Bulk properties}

Directly from the Bethe ansatz equations for spin rapidities the (linear) integral equation for the groundstate density function $\rho(v)$ is derived $[4,32]$

$$
\rho(x)=\rho_{0}(x)-\int_{-K}^{K} k(x-y) \rho(y) d y,
$$

where the so-called bare density $\rho_{0}$ and the kernel $k(x)$ are defined by

$$
\rho_{0}=\frac{1}{2 \pi} \frac{2}{x^{2}+1}, \quad k(x)=\frac{1}{2 \pi} \frac{4}{x^{2}+4} .
$$

Here we have as yet left unspecified the magnetization $m$ of the state (or equivalently the external magnetic field $h$ ) corresponding to some integration terminal $K$ with subsidiary condition

$$
m=\frac{1}{2}-\int_{-K}^{K} \rho(x) d x, \quad 2 k_{F}=(1-2 m) \pi,
$$

where (twice) the Fermi momentum $k_{F}$ is the momentum at which elementary spin excitations become soft. In terms of the density function the groundstate energy $e$ takes the form

$$
e=\int_{-K}^{K} \epsilon_{0}(x) \rho(x) d x,
$$

where $\epsilon_{0}(x)$ is the bare energy of spin excitations

$$
\epsilon_{0}(x)=-\frac{4 J}{x^{2}+1}, \quad \xi_{0}(x)=1,
$$

and the bare charge function $\xi_{0}(x)$ has been introduced for later purposes.

In the same way as (27) defines a function $\rho$ for the inhomogeneity $\rho_{0}$, it gives rise to the dressed energy $\epsilon(x)$ and the dressed charge $\xi(x)$ related to (31). (Note that the auxiliary function $\mathfrak{a}(x)$ of the thermodynamic treatment is related to $\epsilon(x)$ in the lowtemperature limit via $\log \mathfrak{a}=\beta \epsilon$ and (3) turns into the linear integral equation for the dressed energy.) As the integration operator in (27) is selfadjoint we have an alternative relation to (30) and (29) for the groundstate energy $e$ and magnetization $m$

$$
e=\int_{-K}^{K} \epsilon(x) \rho_{0}(x) d x, \quad m=\frac{1}{2}-\int_{-K}^{K} \xi(x) \rho_{0}(x) d x .
$$

For relating the terminal $K$ to the magnetic field $h$ we note that the bare energy in the presence of an external field is given by $\epsilon_{0}(x)+h=\epsilon_{0}(x)+h \xi_{0}(x)$. Hence the corresponding dressed energy is $\epsilon(x, h)=\epsilon(x)+h \xi(x)$. A general property of the dressed energy is that it has to vanish at $K$ yielding

$$
\epsilon(K, h)=0 \Leftrightarrow h=-\frac{\epsilon(K)}{\xi(K)} .
$$


For any value of $K$ the functions $\epsilon(x), \xi(x)$ can be evaluated (at least numerically) from an integral equation of type (27), then the magnetic field $h$, and the corresponding magnetization $m$ and groundstate energy $e-m h$ can be calculated utilizing (33) and (32).

\section{Correlation functions}

We are particularly interested in the determination of scaling dimensions (critical exponents) for arbitrary external fields. For instance the spin-spin and energy-energy correlations decay algebraically with exponents $x_{s}$ and $x_{e}$

$$
\left\langle\vec{S}_{0} \vec{S}_{r}\right\rangle \simeq \frac{(-1)^{r}}{r^{2 x_{s}}}, \quad\left\langle\epsilon_{0} \cdot \epsilon_{r}\right\rangle \simeq \frac{\cos \left(2 k_{F} r\right)}{r^{2 x_{e}}}+\frac{C}{r^{2}},
$$

where the energy operator is $\epsilon_{r}=\vec{S}_{r} \vec{S}_{r+1}$. In general the exponents are directly given in terms of the dressed charge function at the terminal $K$ (see [32])

$$
x=\frac{1}{4 \xi(K)^{2}} S^{2}+\xi(K)^{2} d^{2},
$$

where $S$ and $d$ are integers corresponding to the quantum numbers of the particular operator (occurring in the correlation function); $S$ equals the magnetization and $d$ equals the lattice momentum in units of $2 k_{F}$. For spin and energy operators (correlations) we have $(S, d)=(1,0)$ and $(S, d)=(0,1)$, respectively. Therefore we obtain in terms of the dressed charge

$$
x_{s}=\frac{1}{4 \xi(K)^{2}}, \quad x_{e}=\xi(K)^{2} .
$$

We want to note that (35) is obtained from predictions by conformal field theory about finite-size effects for the spectrum of the system on large lattices $L$ with periodic boundary conditions

$$
E_{x}-E_{0}=2 \pi \frac{v}{L}(x+\delta(L))
$$

where $E_{0}$ is the groundstate energy, $E_{x}$ the low-lying energy level corresponding to the operator with dimension $x, v$ the velocity of the elementary excitations, and $\delta(L)$ is a function with zero limit for $L \rightarrow \infty$. The evaluation of (37) can be carried out analytically yielding (35) (see for instance [32] and references therein). Of course, the dressed charge $\xi$ has to be calculated numerically. Alternatively, similar results can be obtained by numerical calculations on the basis of the Bethe ansatz equations for finite systems and (37), however involving an explicit extrapolation analysis with the usual disadvantages, see [33] and for finite external fields [34]. 


\section{Perturbations of the pure system}

The algebraic decay of the correlation functions in the groundstate of the spin- $1 / 2$ Heisenberg chain constitutes quantum criticality. Starting from this observation we are going to argue that the response of the system to perturbations can be understood and evaluated in a renormalization group framework and in general shows non-integer exponents (in contrast to simple perturbation treatments which of course are not legitime). We want to examine the critical exponents of the Spin-Peierls transition which is a structural transition of the underlying lattice with a modification of the local exchange couplings proportional to the local lattice distortion

$$
H(\delta)=H+\sum_{j} \delta_{j} \cdot \vec{S}_{j} \vec{S}_{j+1}=H+\delta H .
$$

An application of scaling relations will provide a simple tool to understand some of the essential aspects of the theory of $[16,17]$. We remind the reader of the response of the free energy $f$ and the correlation length $\xi$ of a critical, classical system in $d$ dimensions perturbed by a relevant operator with RG eigenvalue $y(>0)$

\begin{tabular}{|c|c|c|}
\hline $\begin{array}{l}\text { classical system } \\
d \text { dimension }\end{array}$ & $\longrightarrow$ & $\begin{array}{l}\mathcal{H}(\delta)=\mathcal{H}+\delta \cdot \mathcal{H}^{\prime} \\
\text { free energy } \Delta f \sim \delta^{\frac{d}{y}} \\
\text { corr. length } \xi^{-1} \sim \delta^{\frac{1}{y}}\end{array}$ \\
\hline
\end{tabular}

A quantum critical system in $d$ dimensions behaves very much the same as it is equivalent to a $d+z$ dimensional classical system, where $z$ is the dynamical critical exponent.

\begin{tabular}{|l|l|}
\hline quantum critical system \\
$d$ dimension \\
g.s. energy $e$, gap $m_{0}$
\end{tabular}$\longleftrightarrow \begin{aligned} & \text { classical system } \\
& d+z \text { dimension } \\
& f, \xi^{-1}\end{aligned}$

For conformally invariant chains we have $d=z=1$. Furthermore the RG eigenvalue $y$ is related to the scaling dimension $x$ of the particular operator by

$$
x+y=d+z=2 .
$$

Now equating the above relations we obtain for the response of the quantum chain in the ground state energy per site $e$ and the excitation gap $m_{0}$ ( $=0$ at the unperturbed point):

$$
\Delta e \simeq-\delta^{\frac{2}{2-x}}, \quad m_{0} \simeq \delta^{\frac{1}{2-x}} .
$$

For the spin-Peierls transition we have to use $x=x_{e}=\xi(K)^{2}$. In Fig. 6 the value of the groundstate exponent $\alpha=2 /\left(2-x_{e}\right)$ is depicted and shows a variation from the value $4 / 3$ for zero external field to 2 at the saturation field. As the exponent is less than 2 the gain in magnetic energy always wins in competition with the loss in elastic energy which is of order $\delta^{2}$ resulting in a structural instability, cp. Fig. 7. Of course this result is completely consistent with $[16,17]$ where bosonization results were used for the energyenergy correlations of the spin- $1 / 2 X X Z$ chain in vanishing external field in combination 


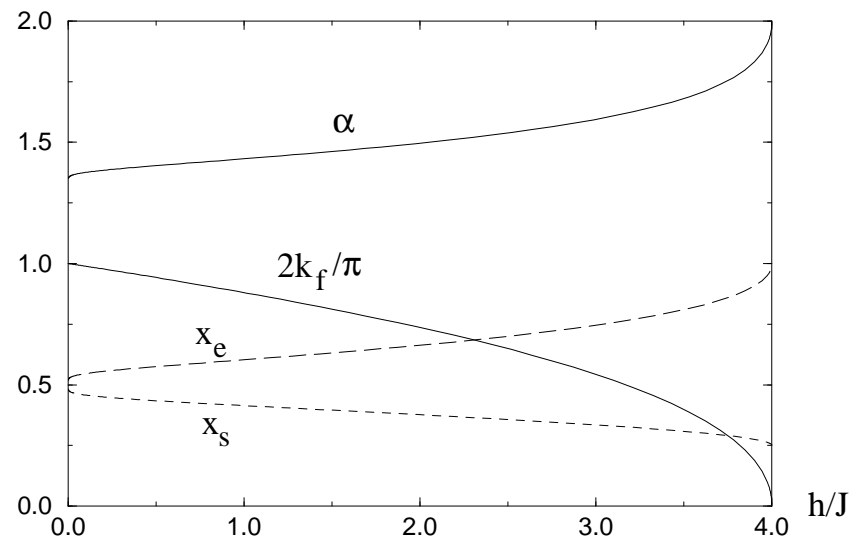

Figure 6: Depiction of scaling dimensions $x_{s}, x_{e}$, groundstate energy exponent $\alpha$, and Fermi momentum $k_{F}$.

with scaling relations for the polarizability. In our treatment we have correctly accounted for the field dependence of the exponents which is new.

Our study is partly motivated by the general interest in a deeper understanding of the physics of Spin-Peierls systems. Here we have shown how a combination of established results by conformal field theory, RG theory and integrable systems yields the exact critical exponents. At the same time we intend to use our results for a more precise calculation of for instance the phase diagram of Spin-Peierls systems in dependence on temperature and magnetic field. Note that there are quantitative discrepancies in for instance the phase boundary between the incommensurate and the uniform phase as obtained experimentally $[35,36]$ and theoretically [17], cf. the remark in [36]. In addition, we note that the occurence of a gap in the magnetic system (for fixed modulation of the exchange couplings) is a very natural consequence of our approach. Experimentally it is still an open question if a spin gap in the incommensurate phase exists. The $T^{3}$ contributions to the specific heat at low temperatures $[37,38]$ are explained in terms of gapless "phasons" [39].

Up to now we have not specified any particular dependence of the perturbation field $\delta_{j}$ on the site index $j$. The above argument (based on a relevant perturbation) is applicable just for the case of a simple algebraic decay of $\left\langle\left(\delta_{i} \epsilon_{i}\right)\left(\delta_{j} \epsilon_{j}\right)\right\rangle$ for $(i-j) \rightarrow \infty$ without any oscillations which imposes a matching condition: (i) the modulation is sinusoidal $\delta_{j}=\delta \cos (q j)$, and (ii) the wave number $q$ is identical to that of the energy-energy correlation $q=2 k_{F}$. (For sinusoidal modulations with different wave number the response in the groundstate energy has the exponent 2.)

As is indicated by the results in Fig. 6 the external magnetic field leads to a filling of the spinon-Fermi sea and a change of exponents $x=x(h)$ and Fermi momentum $k_{F}=k_{F}(h)$. Consequentially an increase in $h$ amounts to (i) a suppression of the SP-instability and (ii) an incommensurate lattice distortion (at least for sufficiently strong fields depending 


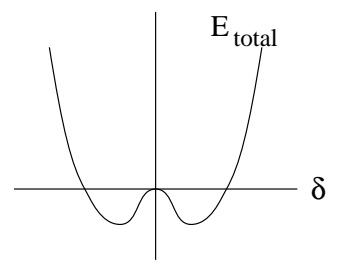

Figure 7: Competition of gain in magnetic energy with loss in elastic energy $E_{\text {total }}(\delta)=$ $E_{\text {mag }}+E_{\text {elast }}=-c_{1} \delta^{\alpha}+c_{2} \delta^{2}$.

on the balance of energy gain in the commensurate versus incommensurate case). These qualitative features are observed in $[35,36]$. Let us consider in some detail the response of the magnetic system (for small magnetization $m$ ) to a sinusoidal modulation of the exchange couplings (with wavevector $2 k_{F}$ ). We are interested in the effects of Umklapp processes which favour commensurate modulations. For $m=0\left(2 k_{F}=\pi\right)$ we expect $E_{\mathrm{mag}}=-c_{1} \delta^{\alpha}$ and for $m \neq 0\left(2 k_{F} \neq \pi\right)$ only $E_{\mathrm{mag}}=-2 c_{1}(\delta / 2)^{\alpha}=-2{ }^{1-\alpha} c_{1} \delta^{\alpha}$. Hence, the groundstate energy of the total system as a function of the magnetization $m$ shows a jump at $m=0$. This is the origin of a 1st order transition from the commensurate to the incommensurate phase at a finite critical field $h_{c}$. Whether higher harmonics ("solitonic modulations") can actually change the order of the transition from 1 to 2 cannot be answered decisively in this approach.

Lastly, we want to comment on logarithmic corrections to the above results. These corrections typically appear if marginal operators exist, i.e. if there are interaction terms compatible with the usual symmetries of the lattice system (lattice translation, spin rotation), not however with strict conformal invariance in the continuum limit. Furthermore, these interactions have to possess the scaling dimension $x_{m}=2$. From the required symmetry properties only the case $(S, d)=(0,2)$ in (35) is singled out with scaling dimension $4 \xi(K)^{2}=4 x_{e}$. With a glance to Fig.6 we directly see that logarithmic corrections only occur for vanishing external field. For completeness we want to note the physical scenario at this point. The function $\delta(L)$ in (37), which typically decays algebraically now has a leading logarithmic contribution $\delta(L) \simeq b / \log L$. The amplitude $b$ also determines the multiplicative logarithmic corrections [28] to

(i) the two-point correlation function of an operator $o(r)$

$$
\langle o(0) o(r)\rangle \simeq \frac{1}{\left[r(\log r)^{b}\right]^{2 x}}
$$

(ii) the response of the critical system to a (relevant) perturbation with $H^{\prime}=\sum_{r=1}^{L} o(r)$ opening a gap

$$
m_{0} \simeq\left(\frac{\delta}{(\log \delta)^{b x}}\right)^{\frac{1}{2-x}}
$$

where in both cases $x$ is the scaling dimension of the operator $o(r)$ and $\delta$ is the (small) 
coupling parameter. In our case and vanishing external field we have $x=1 / 2$ and $b=3 / 2$ [28] such that $m_{0} \simeq \delta^{2 / 3} /(\log \delta)^{1 / 2}$.

\section{Conclusion}

In this work two aspects of the critical spin-1/2 Heisenberg chain were treated. First, the logarithmic corrections to the leading low-temperature properties in the specific heat and susceptibility have been presented. For this purpose we have developed a systematic expansion of the non-linear integral equation governing the thermodynamics of the system. In physical terms, the low-temperature singularities are caused by the algebraic asymptotics of the $S$-matrix of elementary excitations in dependence on the difference in the spectral parameters. This is consistent with the absence of logarithmic corrections for models with long-range interactions [40]. In addition, numerical results have been presented for the Heisenberg chain down to very low temperatures.

Second, at zero temperature we have treated the perturbation of the Heisenberg chain by inhomogeneous couplings. Quite generally this problem can be solved by use of scaling relations. In our case the Spin-Peierls exponents in presence of an external magnetic field have been determined. We like to note that a similar reasoning is applicable to many questions about critical quantum systems perturbed by additional couplings, e.g. interchain couplings.

In future work we want to combine the two approaches, calculation of finite temperature correlation lengths and exact scaling dimensions, to a more detailed description of the spin-Peierls scenario of the Heisenberg system, e.g the critical lines of the phase diagram.

\section{Acknowledgements}

The author acknowledges valuable discussions with K. Fabricius. The comparison to his numerical data for the thermodynamics of finite systems proved essential in achieving high accuracy in the treatment of the non-linear integral equations. The author is grateful to Wuppertal University where parts of the work were performed. Furthermore, financial support is acknowledged by the Deutsche Forschungsgemeinschaft under grant No. Kl 645/3-1 and support by the research program of the Sonderforschungsbereich 341, Köln-Aachen-Jülich. 


\section{References}

1. H. A. Bethe, Z. Phys. 71 (1931), 205

2. J.D. Johnson, S. Krinsky, and B.M. McCoy, Phys. Rev. A 8 (1973), 2526

3. L. Faddeev and L. Takhtajan, Phys. Lett. A 85, 375, 1981

4. R. J. Baxter, "Exactly Solved Models in Statistical Mechanics", Academic Press, London, 1982.

5. A. A. Belavin, A. M. Polyakov and A. B. Zamolodchikov, Nucl. Phys. B 241 (1984), 333

6. J. L. Cardy, "Phase Transitions and Critical Phenomena, Vol.11", eds. C. Domb and J.L. Lebowitz, Academic Press, London 1988

7. M. Takigawa, N. Motoyama, H. Eisaki and S. Uchida, Phys. Rev. Lett. 76 (1996), 4612

8. T. Böske, K. Maiti, O. Knauff, K. Ruck, M. S. Golden, G. Krabbes, J. Fink, T. Osafune, N. Motoyama, H. Eisaki and S. Uchida, preprint, cond-mat/9709215, 1997

9. M. Arai et al., Phys. Rev. Lett. 77 (1996), 3649

10. S. Takagi, H. Deguchi, K. Takeda, M. Mito and M. Takahashi, J. Phys. Soc. Japan 65 (1996), 1934

11. S. Eggert, I. Affleck and M. Takahashi, Phys. Rev. Lett. 73 (1994), 332

12. K. Okamoto and K. Nomura, Phys. Lett. A 169 (1992), 433

13. K. Nomura and K. Okamoto, J. Phys. Soc. Japan 62 (1993), 1123

14. K. Nomura, Phys. Rev. B 48 (1993), 16814

15. R. E. Peierls, "Quantum Theory of Solids", Oxford University, London, 1955

16. M.C. Cross and D.S. Fisher, Phys. Rev. B 19 (1979), 402

17. M.C. Cross, Phys. Rev. B 20 (1979), 4606

18. A. Klümper, Ann. Physik 1 (1992), 540 ; Z. Phys. B 91 (1993), 507

19. T. Koma, Prog. Theor. Phys. 78 (1987), 1213 ; 81, 783, (1989)

20. M. Takahashi, Phys. Rev. B 43 (1991), 5788 ; Phys. Rev. B 44 (1991), 12382

21. R. Z. Bariev, Theor. Math. Phys. 49 (1982), 1021

22. T. T. Truong and K. D. Schotte, Nucl. Phys. B 220 (1983), 77 
23. J. Suzuki, Y. Akutsu and M. Wadati, J. Phys. Soc. Japan 59 (1990), 2667

24. A. Klümper and M. T. Batchelor, J. Phys. A 23 (1990), L189

25. A. Klümper, M. T. Batchelor and P. A. Pearce, J. Phys. A 24 (1991), 3111-3133

26. C. Destri and H. J. de Vega, Phys. Rev. Lett. 69 (1992), 2313

27. F. Woynarovich and H.-P. Eckle, J. Phys. A 20 (1987), L97

28. I. Affleck, D. Gepner, H. J. Schulz and T. Ziman, J. Phys. A 22 (1989), 511

29. M. Karbach and K.-H. Mütter, J. Phys. A 28 (1995), 4469

30. K. Kawano and M. Takahashi, J. Phys. Soc. Japan 64 (1995), 4331

31. A. Klümper and M. Takahashi, to be published

32. V. E. Korepin, N.M. Bogoliubov, and A.G. Izergin, "Quantum Inverse Scattering Method and Correlation Functions", Cambridge University Press, 1993.

33. F. C. Alcaraz, M. N. Barber and M. T. Batchelor, Ann. Phys. (N.Y.) 182 (1988), 280

34. A Fledderjohann, C Gerhardt, K-H Mutter, A Schmitt, M Karbach, Phys. Rev. B 54 (1996), 7168

35. V. Kiryukhin, B. Keimer, Phys. Rev. B 52 (1996), R704

36. T. Lorenz, U. Ammerahl, T. Auweiler, B. Büchner, A. Revcolevschi, G Dhalenne, Phys. Rev. B 55 (1997), 5914

37. X. Liu, J. Wosnitza, H.v. Löhneysen, and R.K. Kremer, Z. PhysB 98, 163, 1995

38. M. Weiden, J. Köhler, G. Sparn, M. Köppen, M. Lang, C. Geibel, and F. Steglich, Z. Phys. B 98, 167, 1995

39. S.M. Bhattacharjee, T. Nattermann, and C. Ronnewinkel, cond-mat/9711094

40. Y. Kuramoto and Y. Kato, J. Phys. Soc. Japan 64 (1995), 4518 


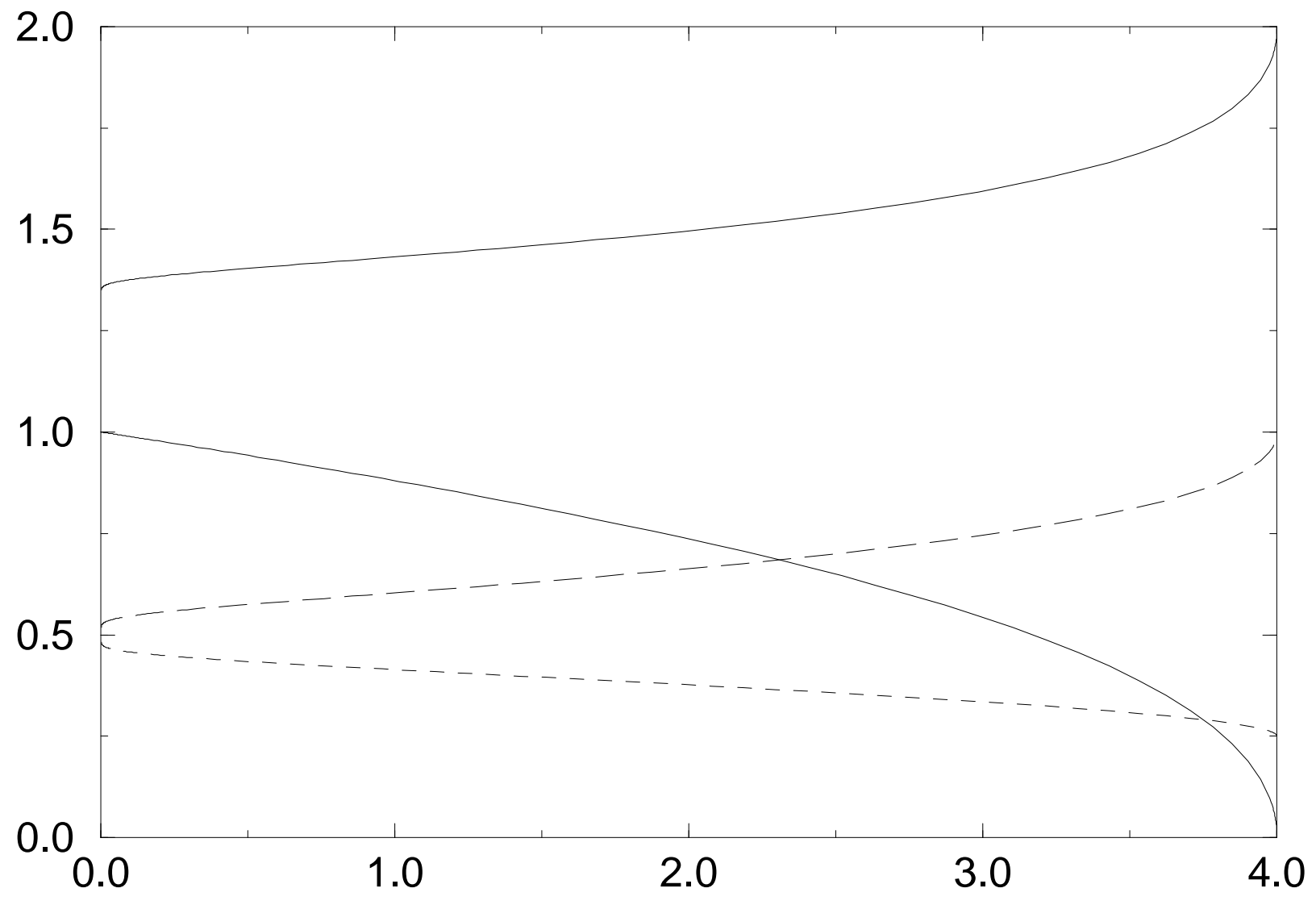

